\section{Análise da distribuição espacial da hanseníase no Estado de São Paulo, 1991-2002}

\section{Analysis of the spatial distribution of leprosy in The State of São Paulo, 1991-2002}

\author{
Paula Araujo Opromolla ${ }^{1}$ \\ Ivete Dalben ${ }^{2}$ \\ Márcio Cardim ${ }^{3}$ \\ 'Instituto Lauro de Souza Lima \\ ${ }^{2}$ Departamento de Saúde Pública. Faculdade de Medicina - FMB/UNESP. \\ Botucatu, SP \\ ${ }^{3}$ Departamento de Matemática, Estatística e Computação. Faculdade de \\ Ciências e Tecnologia - UNESP. Presidente Prudente, SP
}

Trabalho realizado no Departamento de Saúde Pública. Faculdade de Medicina - FMB/UNESP Botucatu, SP.

Correspondência: Paula Araujo Opromolla. Instituto Lauro de Souza Lima, Rod. Comte. João Ribeiro de Barros. km 225.CP 3021 - Bauru, SP CEP:17034-971.E-mail:opromolla@usp.br

\section{Resumo}

A hanseníase no Brasil ainda é um problema a ser equacionado e, no Estado de São Paulo, há varias regiões com altas taxas de detecção. O presente estudo objetivou analisar a distribuição e quantificar a dependência espacial das taxas médias de detecção da hanseníase no Estado de São Paulo, no período de 1991-2002, empregando técnicas geoestatísticas. Verificouse tendência levemente decrescente das taxas médias de detecção para o Estado de São Paulo. Altos índices do indicador podem ser visualizados nas regiões oeste e noroeste de São Paulo. A dependência espacial encontrada foi de aproximadamente $30 \mathrm{~km}$. Com os resultados encontrados, conclui-se que a análise de superfície das taxas médias de detecção pode auxiliar na escolha de áreas prioritárias visando aos exames de coletividade e ao incremento dos exames nos contatos dos casos detectados.

Palavras-chave: Hanseníase. Taxa de detecção. Geoprocessamento. Endemia hansênica. Análise espacial. Geoestatística. Krigagem. 


\section{Abstract}

Leprosy is a problem yet to be solved in Brazil, and in the State of São Paulo there are still areas with high detection rates. The present study aimed to analyze the distribution and quantify the spatial dependency of mean detection rates for leprosy in the State of São Paulo, during the 19912002 period, utilizing geostatistical techniques. A slight tendency toward decreased mean detection rates was verified in the State of São Paulo. High indexes of the indicator could be visualized in the West and Northwest regions of São Paulo. An estimated $30 \mathrm{~km}$ spatial dependency was detected. Based on the results, it could be concluded that analyzing the surface of mean detection rates may be helpful for choosing priority areas for examining groups of people, and to increment tests for the contacts of cases detected.

Keywords: Leprosy. Detection rates. Geoprocessing. Leprosy endemics. Spatial analysis. Geostatistics. Krigage.

\section{Introdução}

A hanseníase é uma doença conhecida há milênios ${ }^{1}$; todavia, apesar de todos os esforços envidados até agora, ainda é um sério problema de saúde pública em alguns países, inclusive no Brasil ${ }^{2}$.

A meta de eliminação da hanseníase como problema de saúde pública, proposta pela Organização Mundial de Saúde em 1991, baseia-se na redução da prevalência da doença para menos de 1 doente por 10.000 habitantes $^{3}$. Entretanto, o coeficiente de prevalência é muito influenciado por aspectos operacionais dos programas de hanseníase e, para o monitoração da endemia, um indicador alternativo e melhor é a taxa de detecção de novos casos ${ }^{4-7}$.

A hanseníase no Brasil ainda é um problema a ser equacionado e, no Estado de São Paulo, há várias regiões com altas taxas de detecção. O estudo do comportamento espacial desse indicador e a avaliação da sua dependência espacial podem ser ferramentas valiosas para auxiliar no planejamento, monitoração e avaliação de ações de saúde, direcionando as intervenções para reduzir as iniqüidades ${ }^{8}$, principalmente os programas de avaliação e de controle da hanseníase no Estado.

O presente estudo objetivou analisar a distribuição e quantificar a dependência espacial das taxas médias de detecção da hanseníase no Estado de São Paulo, no período de 1991-2002, empregando técnicas geoestatísticas.

\section{Metodologia}

Foi conduzido um estudo ecológico da variabilidade espacial das taxas de detecção média da hanseníase, tendo como unidade de análise os municípios.

As taxas médias de detecção foram obtidas com dados de série histórica de 12 anos dos registros dos casos de hanseníase no Estado de São Paulo, contidos nos arquivos informatizados do Centro de Vigilância Epidemiológica da Secretaria de Estado da Saúde de São Paulo (CVE-SP), 
segundo a Ficha de Notificação de Hanseníase do Sistema de Informação de Agravos de Notificação (SINAN), e a população total de cada município, observada em 01 de julho de 1996 conforme as projeções obtidas retrospectivamente a partir do Censo Demográfico de $2000^{9}$.

Foram retirados os casos menor ou igual a um ano de idade e aqueles que, apesar de terem sido notificados no Estado de São Paulo, residiam em outros Estados.

Foram incluídos no estudo 606 municípios do Estado de São Paulo que possuíam ao menos um caso de hanseníase detectado no período.

A análise geoestatística é utilizada para detecção de variabilidade espacial do objeto de estudo em um campo amostral. Doenças transmissíveis, como a hanseníase, apresentam padrão espacial no seu comportamento.

Krige $^{10}$ verificou, experimentalmente, estudando dados de concentração de ouro que, se fossem levadas em consideração as distâncias de amostragem, as variâncias passavam a fazer sentido, não obtendo os mesmos resultados quando as considerava independentes. Baseado nestas informações, Matheron ${ }^{11}$ formalizou os conceitos fundamentais da geoestatística, conhecida como teoria das variáveis regionalizadas, tendo como base os conceitos de função aleatória e estacionariedade de segunda ordem.

Uma função aleatória $Z\left(x_{i}\right)$ é estacionária de ordem 2 se:

- o valor esperado $E\left[Z\left(x_{i}\right)\right]$, existir e não depender da posição $x_{i}$, ou seja, $E\left[Z\left(x_{i}\right)\right]$ $=m$, onde $m$ é uma constante (estacionariedade do primeiro momento estatístico);

- para cada par de variáveis aleatórias $\left.\left\{Z\left(x_{i}\right), Z\left(x_{i}\right)+h\right)\right\}$, a função de covariância, $C(h)=E\left[Z\left(x_{i}\right) Z\left(x_{i}+h\right)\right]-m^{2}, \forall x_{i} \in$ $S$ (onde $S$ é um campo amostral), existir e for função de $h$ (estacionariedade da covariância).

Sob a hipótese de estacionariedade de segunda ordem, a covariância e o semivariograma são formas alternativas de ca- racterizar a autocorrelação dos pares $Z\left(x_{i}\right)$ e Z $(x+h)$ separados pelo vetor $h$.

A função de semivariograma é definida por:

$\gamma(h)=\frac{1}{2} E\left[Z\left(x_{i}\right)-Z\left(x_{i}+h\right)\right]^{2}$

Em termos práticos, esta função pode ser estimada por:

$\gamma^{*}(h)=\frac{1}{2 N(h)} \sum_{i=1}^{N(h)}\left[Z\left(x_{i}\right)-Z\left(x_{i}+h\right)\right]^{2}$

onde, $N(h)$ é o número de pares de valores amostrados, $\left(Z\left(x_{i}\right), Z\left(x_{i}+h\right)\right)$, separados por uma distância $h$.

O gráfico $\gamma^{*}(h) \times h$, chamado semivariograma experimental, expressa a variabilidade espacial entre as amostras, sendo uma função que só depende do vetor $h$.

O semivariograma experimental, equação (2), deve ser ajustado por um modelo matemático, permitindo assim estimar valores em locais não amostrados. Nesse estudo, o ajuste se fez pelo modelo exponencial, que segue a equação (3).

$\gamma(h)=c_{0}+c\left[1-\exp \left(-3 \frac{h}{a}\right)\right] ; 0<h<d$

onde, $d$ é a máxima distância na qual o semivariograma está definido.

$\mathrm{O}$ ajuste ocorre determinando-se os parâmetros envolvidos no modelo: a distância da dependência espacial $(a)$, conhecida como "range" ou alcance da dependência espacial; o efeito pepita $\left(c_{0}\right)$, conhecido como "nugget effect", que é o valor da semivariância a distância zero; e a altura $c$, distância entre o efeito pepita e o patamar, também conhecida como "sill", que é o intervalo no qual o semivariograma cresce. O patamar representa a altura em que o semivariograma se estabiliza, aproximando-se da variabilidade total dos valores amostrados.

Havendo dependência espacial, verificada pelo semivariograma, e levando-se em consideração as hipóteses de variância mínima e não tendenciosidade podem ser estimados, pela interpolação por krigagem, 
valores da variável em estudo em locais onde ela não foi amostrada.

A krigagem é um método de estimativa semelhante às médias móveis com valores distribuídos no espaço a partir de valores adjacentes, enquanto esses valores são considerados interdependentes pela função da semivariância ${ }^{12}$.

As estimativas são calculadas pela equação 4 .

$$
Z^{*}\left(x_{0}\right)=\sum_{i=1}^{N} \lambda_{i} Z\left(x_{i}\right)
$$

onde

- $Z^{*}\left(x_{0}\right)$ é o valor estimado no ponto $x_{0}$;

- $\quad N$ é o número de pares de valores medidos $Z\left(x_{i}\right)$, envolvidos na estimativa;

- $\lambda_{i}$ são os pesos associados a cada valor medido $Z\left(x_{i}\right)$.

Os pesos, na equação 4 de krigagem, são determinados minimizando-se a variância: $\operatorname{Var}\left[Z^{*}\left(x_{0}\right)-Z\left(x_{0}\right)\right]=E\left[Z^{*}\left(x_{0}\right)-\right.$ $\left.Z\left(x_{0}\right)\right]^{2}$, sujeitos a restrição de não tendenciosidade: $E\left[Z^{*}\left(x_{i}\right)-Z\left(x_{0}\right)\right]=0$.

A condição de não tendenciosidade mostra que não existe diferença entre valores estimados e amostrados, pois mesmo os valores amostrados serão estimados pela equação de krigagem, utilizando-se pesos tais que sua soma seja igual a um ${ }^{13}$.

Levando-se em consideração as hipóteses de variância mínima e não tendenciosidade, chega-se ao sistema de equações de krigagem em termos de semivariância.

$$
\left\{\begin{array}{l}
\sum_{j=1}^{N} \lambda_{j} \gamma\left(x_{i}, x_{j}\right)+\mu=\gamma\left(x_{i}, x_{0}\right) ; \quad i=1, \ldots, N \\
\sum_{j=1}^{N} \lambda_{j}=1
\end{array}\right.
$$

e a variância da estimativa $\sigma_{K}^{2}\left(x_{0}\right)$ é dada pela equação (6):

$\sigma_{K}^{2}\left(x_{0}\right)=\mu+\sum_{j=1}^{N} \lambda_{i} \gamma\left(x_{i}, x_{0}\right)$

O erro da estimativa pode ser avaliado pelo processo denominado de validação cruzada, que consiste em estimar valores em cada ponto medido. Realiza-se esta estimativa excluindo-se um ponto do conjun- to original de dados e estima-se o valor da variável para aquele ponto a partir dos restantes, utilizando-se a krigagem. Retornase o ponto ao conjunto e retira-se o seguinte, repetindo o processo para todos os pontos amostrados. Para cada ponto, é possível então obter o erro de estimação e padronizá-lo pelo desvio padrão da estimação. Espera-se que o conjunto dos erros padronizados tenha distribuição normal de média zero e variância unitária. O erro da estimativa é dado por: $E\left(x_{i}\right)=Z\left(x_{i}\right)-\hat{Z}\left(x_{i}\right)$, sendo que a média dos erros é próxima de zero: $\frac{1}{n} \sum_{i=1}^{n}\left[Z\left(x_{i}\right)-\hat{Z}\left(x_{i}\right)\right] \cong 0$ e a média do quadrado dos erros padronizados está próxima de um: $\frac{1}{n} \sum_{i=1}^{n} \frac{\left[Z\left(x_{i}\right)-\hat{Z}\left(x_{i}\right)\right]^{2}}{s_{E}^{2}\left(x_{i}\right)} \cong 1$, onde o desvio padrão de krigagem $s_{E}\left(x_{i}\right)$ representa o erro do valor estimado pelo modelo quando a krigagem é feita para a localização $x_{i}$ (omitindo-se o valor amostral da localização $x_{i}$ ). O gráfico dos erros $\left(E\left(x_{i}\right)\right)$ "versus" os valores estimados $\left(\hat{Z}\left(x_{i}\right)\right)$ deverá ser centrado ao redor da linha zero. Além disso, o gráfico deverá ter uma dispersão por igual, isto é, a variância do erro deverá depender da grandeza do valor. Se os valores estimados forem idênticos aos medidos, o coeficiente de correlação será de $100 \%$.

A formatação dos bancos de dados do CVE-SP e as análises estatísticas foram realizadas com o software SPSS ${ }^{14}$. Selecionaram-se as variáveis ano de registro e município de residência dos casos de hanseníase.

Para as análises geoestatísticas, cálculo das semivariâncias e dos parâmetros do modelo matemático ajustado, utilizou-se o software GS+ for Windows 5.0.3 Beta ${ }^{15}$.

O software Surfer ${ }^{16}$ possibilitou a interpolação pela krigagem e as estimativas das taxas de detecção de hanseníase, que permitiram construir os mapas de risco das taxas de detecção de hanseníase no Estado de São Paulo.

\section{Resultados}

Dos 645 municípios existentes no Es- 
tado de São Paulo, no período estudado, em 606 deles foi notificado pelo menos um caso de hanseníase. As estatísticas descritivas básicas das taxas médias de detecção estão apresentadas na Tabela 1.

A Figura 1 apresenta a evolução temporal dos coeficientes de detecção da
Hanseníase, por 10.000 habitantes, no Estado de São Paulo, entre 1991 e 2002.

A idade média dos casos estudados foi de 43 anos ( \pm 17 ); $58 \%$ eram do sexo masculino, $57 \%$ classificados como multibacilares, $31 \%$ avaliados com algum grau de incapacidades e $11 \%$ sem avaliação de in-

Tabela 1 - Estatística descritiva das taxas médias de detecção da hanseníase, Estado de São Paulo, 1991-2002.

Table 1 - Descriptive statistics of mean detection rates for leprosy, State of São Paulo, 1991-2002.

\begin{tabular}{|c|c|c|c|}
\hline & & Estatística & Erro padrão \\
\hline Média & & 0,920 & 0,038 \\
\hline Intervalo de confiança & Limite inferior & 0,846 & \\
\hline de 95\% da média & Limite superior & 0,994 & \\
\hline Mediana & & 0,656 & \\
\hline Variância & & 0,925 & \\
\hline Desvio padrão & & 0,962 & \\
\hline Mínimo & & 0,00 & \\
\hline Máximo & & 11,70 & \\
\hline Amplitude & & 11,70 & \\
\hline Coeficiente de variação & & 0,767 & \\
\hline Coeficiente de assimetria & & 3,965 & 0,096 \\
\hline Coeficiente de curtose & & 29,290 & 0,192 \\
\hline
\end{tabular}

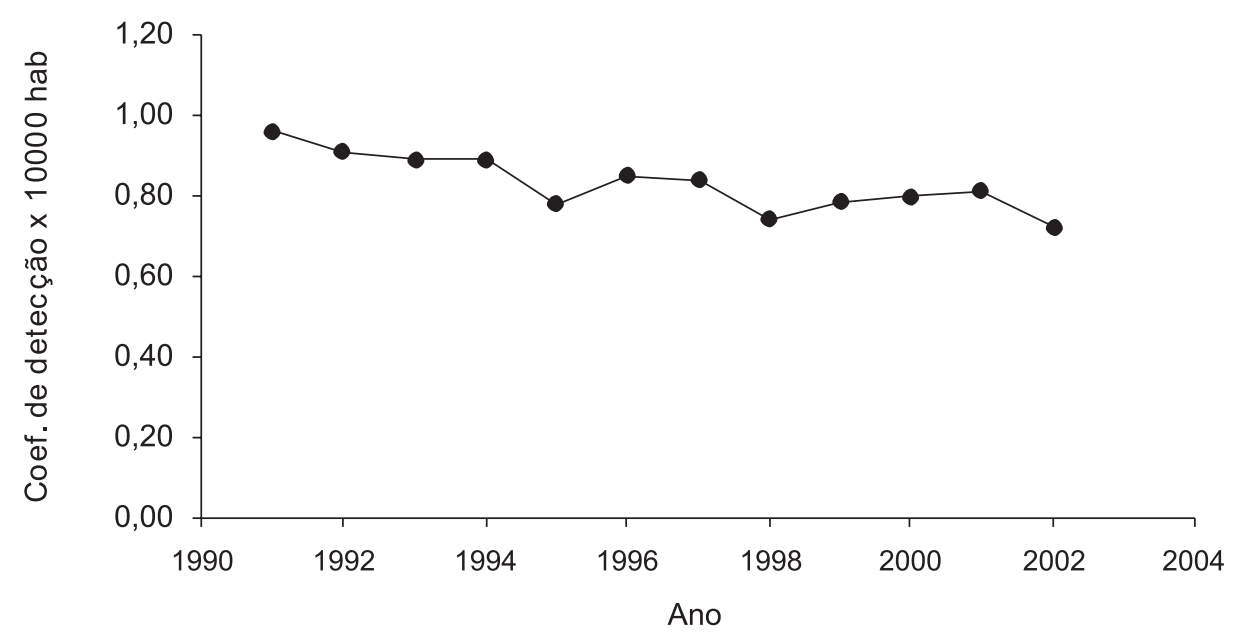

Fonte:SES/CVE - Divisão Técnica Vig. Epid. Hanseníase dados atualizados em: 05/04/05

Figura 1 - Evolução temporal dos coeficientes de detecção da Hanseníase, por 10.000 habitantes, no Estado de São Paulo, 1991-2002.

Figure 1 - Time trend for leprosy detection coefficients per 10.000 inhabitants, State of São Paulo, 1991-2002. 
capacidades. Quanto ao modo de detecção, apenas $8,2 \%$ se devem a exame de contatos e de coletividade.

Com a análise geoestatística, ajustando-se um modelo exponencial, a confecção do semivariograma experimental apresentou os seguintes parâmetros: $\mathrm{C}_{0}=0,097$, $\mathrm{C}_{0}+\mathrm{C}=0,306$ e $\mathrm{a}=0,29$.

Nesse estudo, o alcance foi de 0,29 graus de coordenadas geográficas que correspondem aproximadamente a $30 \mathrm{~km}$.

A Figura 2 mostra o semivariograma para as taxas de detecção da hanseníase e seu ajuste por meio do modelo Exponencial.

As estimativas das semivariâncias em função da distância e o modelo ajustado a elas foram mostrados graficamente para facilitar a visualização e interpretação da variabilidade espacial.

A validação do modelo foi realizada pelo processo de validação cruzada.

Encontrados valor do coeficiente de determinação $r^{2}=0,20$ e valor próximo de um para o coeficiente de regressão $(0,803)$, esses indicam que houve bom ajuste para a função exponencial, descrevendo, assim, uma eficiente relação entre os valores es- timados e os observados.

No gráfico da validação cruzada estão representados os valores estimados por krigagem "versus" os valores observados das taxas de detecção de hanseníase analisadas. Podem-se observar duas características que são desejáveis na validação cruzada: os pontos devem estar acompanhando a diagonal traçada (reta estimada pela regressão) o mais próximo possível; os dois lados da diagonal devem estar equilibrados (Figura 3).

Com a confecção dos semivariogramas direcionais em $0^{\circ}, 45^{\circ}, 90^{\circ}$ e $135^{\circ}$, é possível afirmar que o fenômeno estudado é isotrópico, uma vez que, independentemente da direção, os semivariogramas permaneceram próximos um do outro (Figura 4).

$\mathrm{O}$ ajuste do semivariograma e a interpolação realizada pela krigagem ordinária, com 10.000 pontos interpolados, possibilitaram a confecção do mapa de isolinhas, que expressam, em termos de probabilidade, áreas com maior ou menor risco para a doença, representadas na Figura 5, pelo mapa de isolinhas.

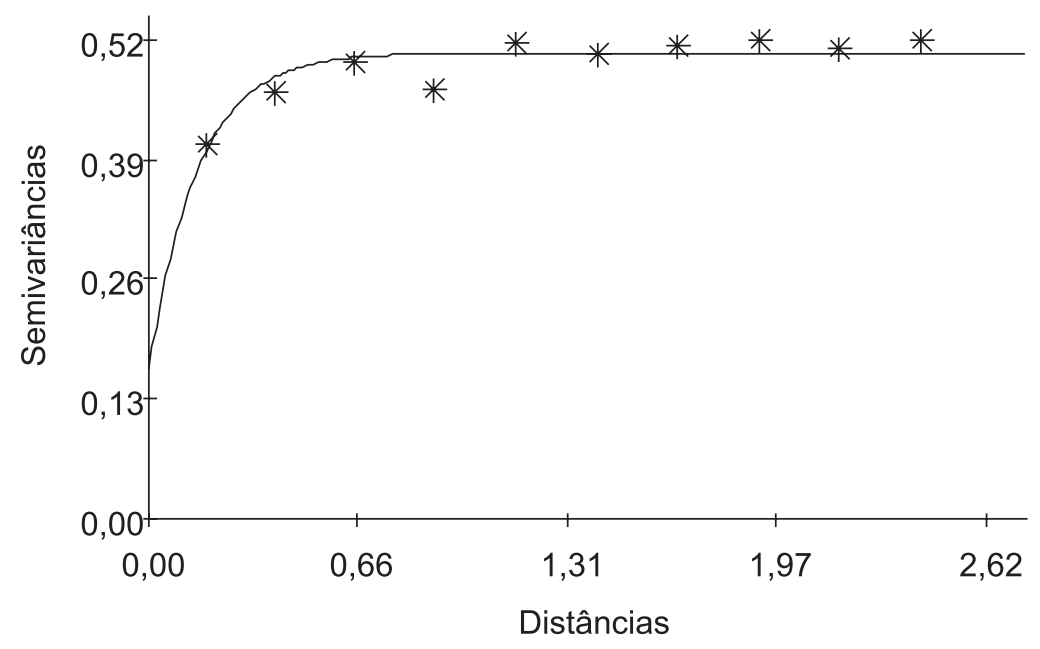

Obs: Distâncias em graus de coordenadas geográficas

Figura 2 - Semivariograma das taxas de detecção da hanseníase ajustado por meio do modelo Exponencial.

Figure 2 - Semivariogram of the detection rates of leprosy adjusted by means of the Exponential model. 


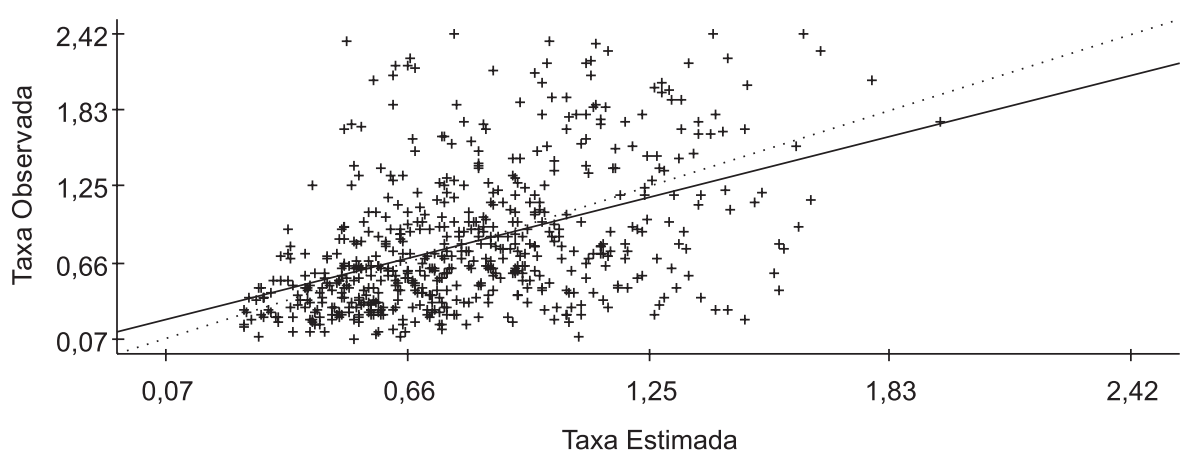

Figura 3 - Validação do modelo ajustado, com a representação dos valores estimados por krigagem "versus" os valores observados das taxas de detecção de hanseníase analisadas.

Figure 3 - Validation of the adjusted model with representation of the values estimated by krigage "versus" observed values of analyzed leprosy detection rates.

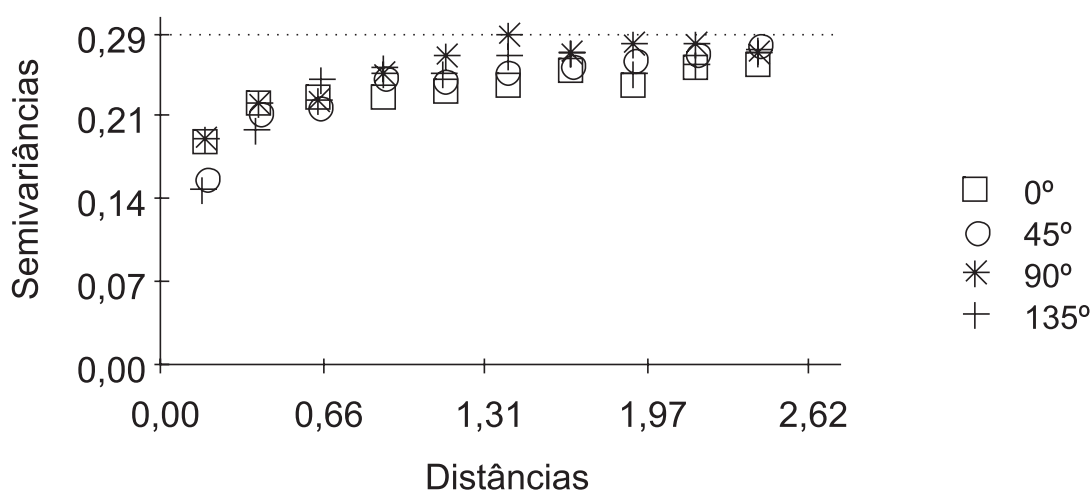

Obs: Distâncias em graus de coordenadas geográficas

Figura 4 - Semivariogramas direcionais das taxas médias de detecção da hanseníase.

Figure 4 - Directional semivariograms of mean leprosy detection rates.

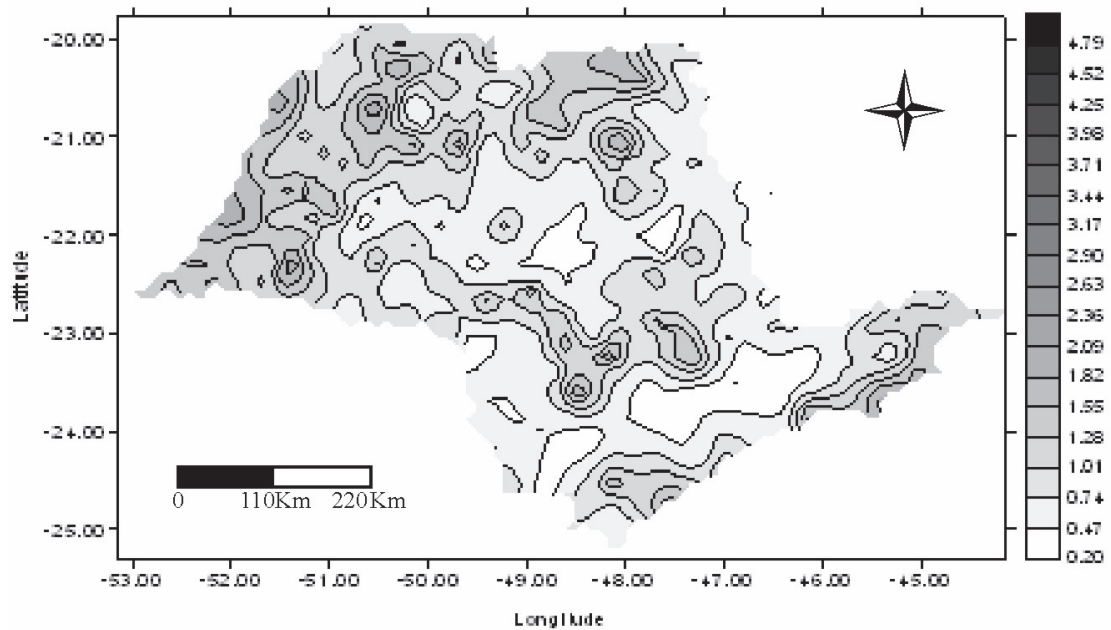

Figura 5 - Espacialização das taxas de detecção de hanseníase, no Estado de São Paulo, 19912002.

Figure 5 - Spatialization of leprosy detection rates in the State of São Paulo, 1991-2002. 


\section{Discussão e Conclusão}

Em São Paulo, o comportamento de detecção de casos de hanseníase vem se mantendo constante nos últimos 30 anos. Estudos de tendência para as taxas de detecção no período de 1969 a 1985 mostraram um crescimento de $1 \%$ ao ano no Estado de São Paulo ${ }^{17}$; outro estudo, realizado no período de 1982 a 1992, mostrou leve decréscimo do indicador ${ }^{18}$, semelhante ao encontrado neste estudo, como pode ser visto na Figura 1.

Os coeficientes de detecção são indicadores de transmissibilidade da infecção ${ }^{19}$. No entanto, a redução desse indicador deve ser vista com cautela, pois essa diminuição pode ser efeito da substituição da detecção ativa pela passiva de casos, pela falta de conscientização dos profissionais e da comunidade, ou mesmo de diagnóstico errado.

A distribuição da hanseníase no Estado de São Paulo, como em todo o Brasil, ocorre de maneira desigual entre as áreas. Dessa forma, é necessário que os gestores conheçam a situação epidemiológica local para definirem as ações prioritárias de acordo com cada situação.

Segundo Mencaroni (2003), uma das preocupações atuais é em relação aos 48,8\% dos municípios do Estado que apresentam coeficientes de detecção da doença nulos. Esse silêncio epidemiológico pode ser entendido como uma nova realidade epidemiológica na região ou ausência de ações de busca ativa de casos novos ${ }^{20}$.

A análise espacial da superfície do Estado de São Paulo, utilizando-se a geoestatística, no caso da hanseníase, parece ser uma abordagem que pode auxiliar o reconhecimento de áreas de risco dando suporte a estratégia de controle e eliminação da doença, pois evita o particionamento artificial da região, baseado em critérios político-administrativos.

No entanto, essa ferramenta de análise necessita de informações fidedignas, tanto de bases cartográficas como em relação aos agravos, neste caso, da hanseníase.
Uma das dificuldades encontradas neste estudo é a provável inconsistência do banco de dados das notificações de hanseníase. O banco informatizado é aberto e descentralizado, o que proporciona possibilidade de alterações nos dados nos níveis mais primários, que aumentam os problemas de duplicação de registros. A escolha de um período de 12 anos para as análises aqui apresentadas pôde suavizar os efeitos de introdução de novas estratégias de tratamento e controle da doença e as conseqüentes variações das informações anuais.

Outro problema foi a inclusão de localidades que, na metade do período estudado, ainda não haviam sido elevadas à categoria de municípios e, dessa maneira, suas populações foram estimadas considerando-se resultantes de modelo de projeção demográfico baseado nos resultados dos Censos Demográficos (Fundação IBGE) e nos indicadores de crescimento calculados a partir das Estatísticas Vitais processadas na Fundação SEADE e referidas em 1 de julho de cada ano. Essa alteração na malha municipal seria mais prejudicial à análise de áreas, que pôde ser controlada pela análise de superfície.

Os resultados encontrados no presente trabalho indicam altas taxas médias de detecção nas regiões oeste do Estado, que devem estar contribuindo para o aparecimento de novos casos nas regiões Centro Oeste do país, como referem Ignotti et al $(2004)^{21}$. Também na região noroeste do Estado observam-se índices elevados desse indicador.

É possível que o comportamento migratório dessas regiões esteja relacionado a esses achados ${ }^{22}$, aumentando não só a presença de casos, mas também de susceptíveis à doença. A hanseníase é fortemente relacionada às condições socioeconômicas ${ }^{23}$, ao crescimento acelerado da população dessas áreas, ao deslocamento de contingente populacional de áreas rurais para as cidades, que pode não ter sido acompanhado de melhoria da estrutura urbana com conseqüente declínio nas condições de vida dessas populações. 
Apesar dos obstáculos encontrados, os resultados do presente estudo podem colaborar na busca da base do iceberg epidemiológico, possibilitando a visuali- zação de áreas onde devem ser priorizados os exames da coletividade e recomendada busca mais acurada de casos, em especial em seus comunicantes.

\section{Referências}

1. Monot M, Honoré N, Garnier T, Araoz R, Coppée JY, Lacroix C et al. On the origin of leprosy. Science 2005: 308 (5724): 1040-2

2. World Health Organization. Weekly epidemiological record. N 34, 2005, 80, 289-96.

3. World Health Assembly 44.9. Elimination of leprosy: resolution of the 44th World Health Assembly. Geneva: World Health Organization; 1991.

4. Andrade VL, Moreira T, Tardim R, Castro A, Sousa A. Campanha de eliminação da hanseníase combinada com a vacina antipoliomelite. An bras Dermatol 1998; 73: 15965.

5. Pereira GFM. Características da hanseníase no Brasil: situação e tendência no período de 1985 a 1996. [dissertação de mestrado] São Paulo: Escola Paulista de Medicina, Departamento de Medicina Preventiva, Universidade Federal de São Paulo; 1999.

6. Smith WC. We need to know what is happening to the incidence of leprosy. Lepr Rev 1997; 68: 195-200.

7. Lockwood DNJ, Suneetha S. Leprosy: to complex a disease for a simple elimination paradigm. Bull of WHO. March 2005, 83 (3): 230-5.

8. Barcelos C, Santos SM. Colocando dados no mapa: a escolha da unidade espacial de agregação e integração de bases de dados em saúde e ambiente através do geoprocessamento. IESUS 1997; 6: 21-9.

9. Fundação Instituto Brasileiro de Geografia e Estatística. Censo demográfico brasileiro de 2000. Rio de Janeiro: Fundação Instituto Brasileiro de Geografia e Estatística; 2001.

10. Krige DG. A statistical approach to some basic mine evaluation problems on the Witwatersrand. JChem Metall Min Soc Afi 1951; 52: 119-39.

11. Matheron G. Principles of geostatistics. Economic Geology 1963; 58 (8): 1246-66.

12. Goovaerts P. Geoestatistics for natural resources evaluation. New York, Oxford University Press, Inc, 1997.
13. Isaaks EH, Shrivastava RM. An Introduction to Applied Geostatistics. New York, Oxford University Press, Inc., 1989.

14. SPSS for Windows. Chicago: SPSS; 2001.

15. GS+ for Windows 5.0.3 Beta: Goestatistics for the environmental sciences. Michigan: Gama Design Software, 2000.

16. Surfer Version 6.01 - Surface mapping system. Golden: Golden Software; 1995.

17. Zuñiga MG. Informe de assessoria al programa de control de la enfermiodad de hansen en el Brasil. Ministério da Saúde Coordenação Nacional de Dermatologia Sanitária; 1987.

18. Nogueira W. Eliminação da Hanseníase: um projeto de avaliação de impacto de uma intervenção em municípios do Estado de São Paulo com prevalência de eliminação. [dissertação de mestrado]. São Paulo: Faculdade de Medicina, Universidade de São Paulo; 2002.

19. ILEP. The interpretation on epidemiological indicators in leprosy. Technical Bulletin. London; 2001

20. Mencaroni D. Análise espacial da endemia hansênica no município de Fernandópolis/SP. [tese de doutorado]. Ribeirão Preto: Escola de Enfermagem, Universidade de São Paulo; 2003.

21. Ignotti E, Rodrigues AM, Andrade VL, Valente JG. Aplicação de métodos de estimativas de hanseníase no Estado de Mato Grosso. Rev Bras Epidemiol 2004; 7(2): 155-66.

22. São Paulo. (Estado). Cadernos do fórum São Paulo Século XXI: Cidadão do Século XXI. Caderno 1,1-51. [série on line] 1999. Disponível em http://www.al.sp.gov.br/ forum/CADERNOS/index.htm. Acessado em 10/01/2005.

23. Helene LMF, Salum MJL. A reprodução social da hanseníase: um estudo do perfil de doentes com hanseníase no Município de São Paulo. Cad Saúde Pública 2002; 18(1): 101-13.

Recebido em: 16/06/05 Versão reformulada reapresentada em: 13/09/05 Aprovado em: 28/09/05 